\section{LIKERT: An APPLESOFT program for the construction, administration, and scoring of Likert scales}

\author{
PERRY R. MORRISON \\ University of New England \\ Armidale, New South Wales 2351, Australia
}

Many of the tasks used in psychological experiments are completely computer controlled. However, before beginning the experiment proper, researchers sometimes wish to preselect subjects or take pretask measures that usually involve the administration of questionnaire material. In addition, experimenters often like to debrief the subject in order to gain the perspective of the individual and to help isolate any flaws or artifacts of design and method. Again, this often takes the form of paperand-pencil questionnaires.

LIKERT consists of two subprograms that allow the experimenter to set up batteries of Likert scale questionnaires and then administer them via a microcomputer and store the scored responses on a floppy disk medium. The first program sets up the questionnaire files, which are administered and scored by the second program. Such a strategy maximizes the amount of random-access memory available for the questionnaire.

Upon running, the first program presents the user with a choice of six modes. Depending on the mode of operation, a file of semantic differential word pairs, a file of questions, or both will have to be constructed. The first mode constructs a file of semantic differential word pairs that are presented beneath Likert scales when the second program is run. Thus in this mode, it is assumed that the subject knows what is being rated (such as the experiment itself) or the experimenter may provide the subject with written material relevant to the adjectives presented when the file is actually executed by the second program. In the second mode, the program constructs both a file of semantic differential word pairs and a file of questions relevant to the words. When the second program is executed in Mode 2, the program presents the scale, a moving cursor, and the words at opposite poles of the linear scale. Because it is impossible to place text within the graphics screen of an unmodified Apple, in this mode the question relevant to that word pair appears in the two lines remaining beneath the high-resolution graphics screen. Thus in this mode, the questions cannot exceed 80 characters (including spaces). For those requiring questions longer than 80 characters, the third mode overcomes this limitation by presenting the question on the text screen and allowing the subject to press a key when he is ready to view the scale (with the relevant word pair) and respond. Questions here may be up to 200 characters in length (including spaces). Modes 4, 5, and 6 differ from the previous three by replacing the semantic differential word pairs with a simple agree/disagree dimension. In Mode 4 , the agree/disagree dimension is presented and, as in Mode 2, questions of fewer than 80 characters are presented beneath the high-resolution screen. The fifth mode allows the user to create and present question files with items of greater length by using the same strategy as Mode 3. That is, the question is presented on the fulltext screen and the subject presses a key when he is ready to respond on the agree/disagree dimension. The last mode simply presents the scale, with its agree/ disagree dimension, and awaits the subject's response. This mode proves useful when the experimenter provides the subject with a written sheet of statements on which the subject is to provide his opinion. In all modes of the set-up program, the user is able to provide names of his choice for the files he is constructing, as well as the name of the data output file. In addition, the number of anchor points for the particular questionnaire can be specified by the user. Thus the scale format can vary from 3 to 13 points.

The second program actually administers the questionnaires and scores the subject's responses. Depending on the mode of operation, it requests the names of the relevant files and reads them into memory. When administration begins, the response scale is presented on the high-resolution graphics screen and the relevant word pair or agree/disagree dimension is placed at the polar extremes. A moving cursor that is controlled by either the Apple game paddles or a joystick plugged into the Apple's game $\mathrm{I} / \mathrm{O}$ is presented slightly below the scale. When the subject has moved the pointer to the position of his choice, he presses the button on the controller, and the Apple emits a beep. The subject's response is scored and stored into an array for later output to a data file already specified by the user. If a subject's response lies between two anchor points, that data point is not lost (as it normally is in paper-and-pencil administration), since the program scores all scales to an accuracy of at least one decimal place. Subsequent scales and items are presented with a delay of less than $2 \mathrm{sec}$.

LIKERT is able to present a maximum of 80 semantic differential word pairs, as well as 80 questions. In Modes 2 and 4, those questions cannot exceed 80 characters; however, in Modes 3 and 5, the question length can be up to 200 characters. If the questionnaires to be administered are greater than 80 items in length, then they can be constructed as a series of smaller files that are presented sequentially by multiple runs of the second program.

Requirements. An Apple II microcomputer with at least $48 \mathrm{~KB}$ of RAM, at least one floppy disk drive, and a game controller or joystick is required.

Language. APPLESOFT BASIC is the language used; conversion to other forms of BASIC would prove 
tedious because of the extensive use of Apple-specific disk commands and high-resolution graphics.

Availability. Program listings and disk copies are available (provided that an unformatted floppy disk is supplied) for $\$ 2$ (to cover costs of copying and/or mailing) from Perry R. Morrison, Department of Psychology, University of New England, Armidale, New South Wales 2351, Australia.

(Accepted for publication December 30, 1982.) 